\title{
Die Suche nach Sicherheit: Kombinierte Produktionsformen im sogenannten Informellen Sektor*
}

\author{
Georg Elwert \\ Hans-Dieter Evers \\ Werner Wilkens \\ Universität Bielefeld, Fakultät für Soziologie \\ Universitätsstrałse 25, D-4800 Bielefeld
}

$\mathrm{Z}$ u s a $\mathrm{m}$ m e $\mathrm{n}$ f a s $\mathrm{s}$ u $\mathrm{n}$ g: In der soziologischen Beschäftigung mit Arbeitslosigkeit in europäischen Ländern wird zunehmend auf Arbeitsformen verwiesen, die sich jenseits von Lohnarbeit finden. Dieser Bereich wird mit dem in der Entwicklungssoziologie seit längerem diskutierten Begriff des ,,informellen Sektors" belegt. Dieser Aufsatz kommt, ausgehend von empirischen Studien in Entwicklungsländern, zu dem Schluß, daß der Begriff informeller Sektor zwar deskriptiv, wohl aber kaum analy tisch verwendbar sei. Der angesprochene Bereich läßt sich soziologisch als "Schicht der Ungesicherten" bezeichnen, in der die Suche nach Sicherheit eine absolute Priorität vor der Einkommensmaximierung hat. Bei dieser Suche nach Sicherheit sticht gerade der Versuch der Schaffung kooperativer Strukturen und der meist vergebliche Appell an den Staat zur Bereitstellung kooperativer Strukturen der Sicherun hervor. Solche Versuche haben bisweilen die Form von ethnischen Vereinigungen oder auch die Form „neuer Kirchen". Die Informalisierung bzw. Peripherisierung eines Teil der Okonomie und Gesellschaft beruht auf einem Einsatz von Macht zur Uberformung des Marktgeschehens. Diese Privilegierungs- und Monopolisierungsprozesse konzentrieren die Ressourcen in einem staatlichen/urbanen/großindustriellen Bereich und entziehen sie so den anderen Sektoren.

In der soziologisch-wissenschaftlichen Beschäftigung mit der Arbeitslosigkeit in der Bundesrepublik wird zunehmend auf Arbeitsformen verwiesen, die sich jenseits von Lohnarbeit finden. Dieser Bereich wird mit dem in der Entwicklungssoziologie seit längerem diskutierten $\mathrm{Be}$ griff des ,informellen Sektors" belegt. Aufgrund einer Durchsicht der Literatur und eigener am Forschungsschwerpunkt Entwicklungssoziologie in Bielefeld durchgefuihrter Felduntersuchungen kommen wir zu dem Schluß, daß der Begriff informeller Sektor deskriptiv, wohl aber kaum analytisch verwendbar ist. Der angesprochene Bereich läßt sich soziologisch als ,Schicht der Ungesicherten" bezeichnen, in der die Suche nach Sicherheit eine absolute Priorität vor der Einkommensmaximierung hat. Das strategische Handeln ist in dieser Sicht auf die Kombination verschiedenartigster Einkommensquellen gerichtet, bei denen der Subsistenzproduktion (Produktion für den Eigenbedarf) besondere Bedeutung zukommt.

Prozesse der Unterentwicklung und strukturierte Reaktionen auf diese Prozesse finden sich nicht nur in der ,Dritten Welt", sondern auch in dem knappen Viertel der Welt, das als industrialisiert gilt. Wenn sich auch die Beispiele des folgenden Aufsatzes im wesentlichen auf (eigene) Forschun. gen in Ländern der Dritten Welt beziehen, so können wir doch auf das Vorhandensein der im folgenden beschriebenen Phänomene und Prozesse auch in industrialisierten Ländern hinweisen (Berger 1982).

\section{Der sogenannte ,informelle Sektor}

Der Begriff des informellen Sektors ist ein Allesfänger, ein catch-all-Begriff. Keith Hart (1973), der illegitime Vater dieses Begriffs ${ }^{1}$, hatte noch

- Dieser Aufsatz geht auf einen Vortrag zum 21. Deutschen Soziologentag zurick. In seiner jetzigen Gestalt haben durch Anregung und Kritik wesentlich beigetragen: Djalma Joana Bezerra, Helmut Fangmann, Carola Lentz, Maria Mies, Tilman Schiel, René Sègbènou, Sundoyo Pitomo, Peter Waldmann und ein anonymer Gutachter der ZrS. Wir danken ganz besonders Christel Huelsewede, die das Manuskript in den verschiedenen Versionen geschrieben hat.

1 Der Ausdruck informeller Sektor findet sich bereits früher, etwa bei Tina Wallace (1972). Als Begriff präzisiert wurde er durch Hart. Freilich sind auch nach Hart noch erhebliche Oszilliationen des $\mathrm{Be}$ griffsinhalts zu finden, so bei Sethuraman, der sich an der Betriebsgröße (unter fünf Beschäftigte) orientiert. Die Diskussion als solche läßt sich unter ande- 
etwas sehr Präzises im Sinn. Er versuchte zu zeigen, daß auch die sogenannten Arbeitslosen in den Armutsbezirken einer Stadt der Dritten Welt (in seinem Beispiel Accra) durchaus Erwerbstätige in den bekannten Produktionsverhältnissen von Lohnarbeit, einfacher Warenproduktion und z.T. auch kapitalistischer Aneignung sind. In einer großartigen Publicity-Kampagne machten eine Reihe von Gutachtern der ILO $^{2}$ hieraus ein Etikett für jenen Bereich der Wirtschaft, der wegen der Schwierigkeiten der Datenerhebung bisher immer vornehm übersehen wurde: den Bereich der Ungesicherten, die durch die Kombination verschiedener wirtschaftlicher Aktivitäten versuchen, die Stabilität zu erlangen, die wir bei einem europäischen Proletarier als selbstverständlich voraussetzen, bzw. aus ökonomischer Sicht gesehen, jenen breiten, armen Rand der Wirtschaft, der kaum von staatlichen Regulationen erfaßt, unterkapitalisiert, trotz größter Anstrengungen aus seinen prekären Bedingungen nicht herauskommen kann. $\mathrm{Zu}$ Recht fragt da eine mexikanische Soziologin, was man denn gewönne, wenn man so verschiedenartige Wirtschaftsaktivitäten wie unterbezahlte Lohnarbeit, Kleinhandel, Subsistenzproduktion (auch Hausarbeit der Frauen) und vielleicht noch die illegalen Varietäten von kapitalistischer Produktion zu einem "Sektor" zusammenfasse (Connolly 1982: 33). Man wirft die extremsten Formen der Ausbeutung ohne Reproduktion der Arbeitskraft zusammen mit aus der Abwehr entstandenen Versuchen, neue autonome Produktionsformen kreativ zu schaffen.

Außerordentlich problematisch erscheint es uns, wenn mit dem Begriff des Sektors suggeriert wird, hier bestünde ein Teil der Ökonomie, der

ren Uberschriften noch länger zurïckverfolgen. Im Rahmen der Dualismus-Theorien entwickelte Geertz (1963) den Begriff der „bazaar-type economy“. Seit Hart findet sich jedoch stärker der Trend, die Verbindungen beider Sektoren zu akzentuieren. Gegen das derzeitige Vergessen dieser Einsicht will auch dieser Aufsatz argumentieren. Nicht zuletzt sei an den holländischen Kolonialökonomen Boeke erinnert, dessen Analyse der Dual-Wirtschaft seit 1930 die wissenschaftliche Diskussion beeinflußt hat (s. Boeke 1981 und die daran anschließenden Kommentare im gleichen Band).

2 Siehe hierzu den Uberblick in Wilkens (1982: 61). Als eine der ersten Publikationen ist zu erwähnen Herbert (1974). Besonders häufig zitiert wurde Sethuraman (1976). weniger Bezüge zu den etablierten ,formellen* Teilen habe als etwa diese unter sich. Verschiedene Untersuchungen zeigen, wie die wesentlichen und tragenden Wirtschaftskreisläufe quer zu dieser hypostasierten Grenze verlaufen; so z.B. die Studie von Santos (1978), in der die Verbindung von Kleinhändlern und kleinen Warenproduzenten (,lower circuit") und Großhandel (,,upper circuit") herausgestellt wird, oder die noch nicht veröffentlichten Untersuchungen von Stauth et al. zur "Straßenökonomie" in Kairo, die Studie von Choe (1982) über Arbeiter in Korea, oder unsere Erhebungen über die Einkommensquellen armer Haushalte in Jakarta (Evers et al. 1982). Die Exportindustrie von Hong Kong wäre ohne den Beitrag der Kontraktfirmen im sogenannten informellen Sektor nicht konkurrenzfähig (vgl. King 1974). Subunternehmer im sogenannten informellen Bereich, Einkommen aus Lohnarbeit in regulären großen Firmen - als ein notwendiger, aber für sich allein nicht überlebenssichernder Beitrag zum Haushaltseinkommen -, der Einbezug von Ressourcen aus dem sogenannten formellen Sektor (Abfallverwertung, illegales Anzapfen von elektrischen Leitungen und Wasserleitungen) konstituieren tragende und nicht marginale Bestandteile des als informell abgegrenzten "Sektors". Illegalität und Illegitimität sollten keinesfalls Merkmale eines ,informeilen Sektors" sein. Opiumschmuggler, Reisschmuggler, Bordelle, Einzelprostituierte, Diebe und Hehler können sowohl gesicherte als auch ungesicherte Existenzen haben, können bei der Allokation von Ressourcen begünstigt sein (2.B. Großbordelle in Bangkok) oder zum peripherisierten Pol gehören. Es versteht sich, daß sie bei einer Klassenanalyse nicht in eine Restkategorie ,Verbrecher" gehören, sondern je nach den Produktionsverhältnissen (Subsistenzproduktion, z.B. bei kleinen Dieben, einfache Warenproduktion, z.B. bei selbst geschaffenen Dienstleistungen wie Prostitution, kapitalistische Produktion, z.B. bei Großhehlern usw.) differenziert werden müssen. Alle diese Tätigkeiten verlaufen quer zu der imaginierten Sektorgrenze.

Diese Beziehungen quer zu der imaginierten Sektorgrenze sind daher nicht nur wesentlich für die Armen, sie sind auch ökonomisch interessant für den formalisierten Bereich der Okonomie. Im Gegensatz zu jenem trickle-downeffect zugunsten der Armen, den sich konserva- 
tive Entwicklungsplaner von Entwicklungsprojekten im formalisierten Sektor versprechen, konnten wir in Untersuchungen in Jakarta einen "trickle-up-effect" beobachten, d.h. eine meßbare Ungleichheit des Tausches, welche die kartellisierten und monopolisierten Kräfte auf dem formalisierten Teil des Marktes begünstigt.

Es ist nun leicht, angesichts dieser Verbindungen des sogenannten informellen oder margina. len Sektors zu den zentralen Bereichen der Ökonomie zu sagen, ,sie dienten dem Prozeß der Kapitalakkumulation" (Kowarick 1982: 22 , ähnlich auch Perlman 1976). Mit dieser Aussage ist aber nur scheinbar etwas gewonnen. Gewiß gibt es für spezifische Kapitale einen besonderen Vorzug in dieser Form der Wirtschaftsverfassung, der ihnen besonders gute Möglichkeiten der Akkumulation einräumt. Ebenso sicher scheint uns aber auch, daß für andere Kapitale eine formalisierte Okonomie (also ohne den ,informellen Sektor") mit überschaubarem Markt unter einer markt wirtschaftlichen Allokation von Produktionsfaktoren, insbesondere von Kapital, welche die Effizienz des gesamten Systems steigern würde, ebenso vorteilhaft oder noch vorteilhafter wäre (vgl. Myint 1971). Die spezifische Differenz dieser Wirtschaftsverfassung wird von diesen Autoren also gerade nicht erfaßt, obwohl sie vorgeben, eine besonders tiefschürfende analytische Einsicht zu verkünden. Eine solche Betrachtung ermöglicht uns, einiges über die Bedeutung des Staates für Länder der Dritten Welt zu erkennen. Allerdings können wir damit nur internationale Vergleiche über mehr oder weniger expansive oder restriktive staatliche Wirtschaftspolitik durchfüren, nicht aber diesen ,,Sektor der Ausgegrenztheit “ international vergleichen. Denn der Umfang und der Inhalt dessen, was ausgegrenzt wird, ist von Land zu Land verschieden. Dies ist auch einer der Gründe dafür, warum lateinamerikanische Studien zum sogenannten informellen Sektor für Forscher in afrikanischen Ländern so eine geringe Attraktivität haben und auch nur bei der Untersuchung weniger asiatischer Länder Berücksichtigung finden konnten.

Der Begriff des informellen Sektors ist also keine analytische Kategorie. Er kann wohl aber eine deskriptive Kategorie sein: Informell ist jener Bereich der Okonomie, der von staatlichem Handeln (einschließlich des Handelns aller staat- lichen Körperschaften) nicht oder unterdurchschnittlich schwach erfaßt wird. Ob dies nun die jeweiligen Autoren einräumen oder nicht, wir können immer wieder feststellen, daß diese direkt oder indirekt durch staatliches Handeln bzw. Nichteingreifen determinierte Grenze konstitutiv ist für den Begriff des informellen Sektors, wie er auf ein spezifisches Land angewendet wird. Hierbei wird oft übersehen, daß diese Grenze je nach politischem System von Land zu Land verschieden ist.

Guisinger und Irfan (1979: 413) geben zu, daß in ihrer Untersuchung die Grenzlinien zwischen formellem und informellem Sektor ,von der Verfugbarkeit von Daten und nicht durch die Theorie diktiert waren". Angesichts dieser Erkenntnis, die wir durch unsere Beobachtungen der Entstehungssituation von Untersuchungen zum informellen Sektor in verschiedenen Län. dern der Dritten Welt bestätigen können, darf es nicht überraschen, wenn der Versuch, diesen Bereich mit statistischen Daten, gewonnen durch die klassischen Erhebungsverfahren von milieufremden Interviewern oder, schlimmer noch, mit Hilfe von statistischen Daten der amtlichen Statistiken zu beschreiben, scheitern muß ${ }^{3}$.

Auch die juristische Grenze zwischen dem Bereich, den der Staat formell erfaßt, und jenem, den er nicht erfaßt und informell beläßt, kann unter Umständen wesentliche Gemeinsamkeiten der Arbeitenden beider Bereiche verhüllen: Choe (1982) wies darauf hin, daß angesichts des Fehlens sozialstaatlicher Elemente in Korea sowohl „,informelle" als auch ein Teil der „formell" BeBeschäftigten in einer Situation der Ungesichertheit leben, also ein wesentliches sozioökonomisches Merkmal gemein haben.

Die Attraktivität des Begriffs ,informeller Sektor kommt jedoch nicht von ungefähr. Tatsächlich korreliert er "irgendwie" mit dem Augenschein. Und an diesem Augenschein ist etwas dran. An den Produktionsverhältnissen läßt sich

3 Man trifft so auf amüsante Ergebnisse wie die einer SUDENE-Studie (1973: 135), die zeigte, daß die Konsumenten des niedrigsten Einkommensbereichs Ausgaben für Lebensmittel hatten, die 113 Prozent (in Worten: einhundertdreizehn) ihres Gesamteinkommens betrugen. 
dieses Besondere - bei aller Mühe der Versuche, diesen Begriff in ein marxistisches Schema zu integrieren - nicht festmachen, denn Lohnarbeiter und einfache Warenproduzenten gibt es diesseits wie jenseits der imaginären Grenze. Selbst die Fluktuation von Arbeitskraft oder die Mischung von Einkommensquellen ist keineswegs auf den sogenannten informellen Sektor beschränkt. Eher schon ist es wichtig und richtig, die Frage zu stellen, warum der Staat diesen oder jenen Bereich der Okonomie nicht erfassen kann oder nicht erfassen will, oder, was nicht übersehen werden soll, warum sich einige Produzenten erfolgreich gegen diese Erfassung wehren können ${ }^{4}$. Diese Auseinandersetzung mit dem Staat zwingt zur Befassung mit den Handlungsstrategien der Betroffenen.

Das, was dem außenstehenden Betrachter unseres Erachtens zu Recht ins Auge springen muß, liegt auf der Ebene der Handlungsstrategien der individuellen Haushalte: der ,Schicht der Ungesicherten " (Elwert 1981; , floating mass", Evers 1980), d.h. der Schicht derer, deren Uberleben nur durch konstantes strategisches Handeln zu sichern ist. Dieses Ziel versuchen sie u.a. durch zweierlei zu erreichen:

\section{1) durch wechselnde Verbindungen ökonomi-} scher Aktivitäten,

2) durch immer neue Bemühungen um kooperative oder korporative Netzwerke der Sicherung.

Bei dieser Schicht ist nach unserer These die Sicherheit nicht etwas strukturell durch staatliche, körperschaftliche oder kooperative Institutionen schon Gegebenes, sondern das immer präsente Ziel strategischen Handelns.

Dabei dürfen kooperative und korporative Institutionen nicht verwechselt oder gar gleichgesetzt werden. Die korporativen Institutionen (in etwa Körperschaften öffentlichen Rechts) sind vom Staat organisiert, geregelt oder $\mathrm{zu}$ mindest in einen rigiden Rahmen eingepaßt. Kooperative (genossenschaftliche, syndikalistische) Institutionen sind demgegenüber egalitä-

4 Der Ausdruck Staat verbirgt je nach historischer Situation verschiedene Inhalte. Die Auseinandersetzungen um die Ausgrenzung oder Eingrenzung bestimmter Bereiche sind konstitutiv für die Arena des Politischen. rere Formen der Selbstorganisation, die oft genug gerade gegen den Staat errungen werden müssen.

Auf der ökonomischen Ebene entspricht diesem Bereich - ohne direkt deckungsgleich mit dem Bereich der Ungesicherten zu sein - die „Peripherie“ der nationalen Wirtschaft, d.h. ein unterkapitalisierter Bereich eines kapitalistischen Nationalsystems, der gerade durch seine Integration in dieses System bei der Zuteilung von Ressourcen benachteiligt ist. Der Prozeß der landesinternen Peripherisierung ist analog zu jenen Prozessen der Peripherisierung, die wir auf Weltmarktebene beobachten können, zu sehen.

Die Suche nach Sicherheit als dominierendes strategisches Handeln und der Ressourcenentzug durch Peripherisierung fuihren zu zwei in der Offentlichkeit sehr ungleich beachteten Prozessen innerhalb des Bereichs der ,Ungesicherten“:

a) Es kommt zu einer Restrukturierung der haushaltsinternen Arbeitsteilung; vor allem zwischen den Geschlechtern. Meist, wenn auch nicht immer, geht dies mit einer besonderen Ausnutzung der Frauenarbeit einher (Werlhof 1981). Entwicklungsstrategien tendieren dazu, dieses interne Verteilungsproblem zu übersehen.

b) Die Entwurzelungsprozesse, die die Schicht der Ungesicherten überhaupt erst konstituieren, hinterlassen beim einzelnen ein Defizit an persönlicher Bedeutung, einen Mangel an Orientierung und eine emotionale Entbehrung. Reaktiv entstehen so kulturelle, religiöse und politische Organisationen, die dem einzelnen Anhänger Geborgenheit, Orientierung und kollektive Größe suggerieren. $^{5}$

5 Beispiele liefern die "grauen Wölfe" in türkischen gecekondus (,slums") und die Khomeini-Bewegung in Iran. Diese Prozesse sind derart wichtig, daB sie eine gesonderte Behandlung verdienen. Aus der klassischen Literatur siehe hierzu Bloch und Mühlmann, zur Reaktion von Emigranten siehe zusammenfassend Elwert 1982. 


\section{Il. Die Schicht der Ungesicherten - Handlungs- strategien auf Haushaltsebene}

Schon lange bevor der informelle Sektor entdeckt wurde, stach denen, die sozioökonomische Analysen furr ihre eigene politische Praxis in Ländern der Dritten Welt durchfürten, eine Gruppe besonders armer Menschen mit nicht klar anggebbarer beruflicher Situation besonders ins Auge. Mao Tse-tung (1926) erfaßte analytisch diese Schicht mit dem Begriff „das Halbproletariat "6 . Dabei dachte er keineswegs an ein Proletariat im Sinne von Lohnarbeit, sondern an die Lohnarbeiter, kleinen Warenproduzenten und an Warenhändler, die sich ihrer SubsistenzReproduktion nicht sicher sein konnten. Diese waren und sind Gelegenheitsarbeiter, Straßenhändler, cash-crop-produzierende Kleinpächter, Gelegenheitsprostituierte usw. Die unvollständige Subsistenz-Reproduktion zwingt sie oft, mehrere Berufe aus diesem Spektrum zu verbinden. $\mathrm{Zu}$ einem Teil trägt auch die Subsistenzproduktion (Produktion für den Eigenbedarf) zum Lebensunterhalt dieser Schicht bei.

\section{Die Sicherung ihrer Subsistenz ist furr sie die} absolute Priorität, die ihr politisches Verhalten bestimmt. Ihr Verhalten geht von klientelistischer Bindung bis zu revolutionärer Mobilisierung. Beispiele für klientelistische Bindung sind die Geheimgesellschaften des vorrevolutionären China (Chesneaux 1971: 57), ähnlich auch jenes Reservoir für Spitzel- und Söldnerdienste für die Franzosen während des Algerien-Kriegs, das Frantz Fanon (1961) als Lumpenproletariat beschrieb. Ein Beispiel für revolutionäre Mobilisierung lieferte Mao Tse-tung, der im Widerspruch zur Mehrheitslinie seiner Partei gerade diese Leute als aktive Kämpfer heranzog, oder

6 Trotz des Titels von Maos Aufsatz ist'seine Analyse soziologisch gesehen keine Klassen-, sondern eine Schichtungsanalyse. Freilich macht sich diese an strukturellen Bedingungen von Uberleben und Risiken fest. Im nächsten Schritt müßte u.E. die Analyse auch zu einer Erfassung "quantifizierter Risiken" kommen. Freilich kann diese Erfassung nicht die klassische Form der ,poverty line" Bestimmung haben; vielmehr ist je nach Erwartungs horizont, Gebrauchswertmustern, Alter, Geschlecht und Haushaltsstruktur zu differenzieren. Die Pluralität der Lebenswelten innerhalb der Ungesicherten legt u.E. nahe, daß ein erster Zugang uber die Analyse der Verarbeitung von Krisen innerhalb von Autobiographien gesucht wird. auch Nito Alves, der die neu zugewanderten Slumbewohner in Angola für die MPLA organisierte. Mit dem (wiederentdeckten) Begriff des Lumpenproletariats, des Halbproletariats bzw. des Protoproletariats haben Fanon und Mao sowie neuerdings McGee die verschiedenen Extreme des politischen Verhaltens dieser Schicht ausgelotet (Armstrong und McGee 1980, Nelson 1969).

Was charakterisiert nun diese sozioökonomischen Gruppen? Sie haben keine gesicherte Reproduktion ihrer Uberlebensbedingungen. Von Berufen, im Sinn des deutschen Wortes, kann man kaum sprechen, da keine einzelne ökonomische Tätigkeit oder keine festgefügte Verbindung mehrerer Tätigkeiten (wie z.B. beim bäuerlichen Mischproduzenten die Verflechtung von Subsistenzproduktion und Warenproduktion) eine langfristige, die Subsistenz sichernde Perspektive geben könnte. Dieses Definitionsmerkmal der "fehlenden Perspektive“, bzw. des „Unzureichenden“ (das auch emischen, d.h. kulturimmanenten Konzeptualisierungen entspricht ${ }^{7}$ ) gewinnt seinen besonderen Sinn durch die Abgrenzung gegenüber der Schicht der gesicherten Lohnarbeiter und einfachen Warenproduzenten. Ihnen gegenüber läßt sich ein soziales Defizit bei der Schicht der Ungesicherten feststellen: Sie haben keine zureichenden kooperativen oder korporativen Sicherungen ihres Lebensunterhalts. Kooperative Sicherungen können etwa Gewerkschaften oder Händlervereinigungen darstellen, korporative Sicherungen bieten Handelskammern, Sozialversicherungen u.ä.

Die Mischung verschiedener Quellen des Haushaltseinkommens, zum Teil aus verschiedenen Produktionsverhältnissen, ist nun nicht nur bei der Schicht der Ungesicherten zu finden. Den reinen Proletarierhaushalt, selbständigen Haushalt usw. gibt es nur als theoretisches Konstrukt. In praktisch jeder Schicht finden wir eine Mischung verschiedener Revenuequellen, insbesondere von Einkommen durch Lohnarbeit, durch einfache Warenproduktion oder einfachen Warenhandel und durch Subsistenzproduktion

7 Entsprechende emische Konzepte sind z.B. „cukupan" (ausreichendes Auskommen) im Indonesischen; das englische Wort ,job", wie es in der französischen Umgangssprache in Cotonou (Bénin) gebraucht wird. 
(Produktion furr den Eigenbedarf) und auch sogar bei der Schicht der Ungesicherten - Einkommen aus Verpachtung oder Vermietung von Grundbesitz und Wohnraum. Diese verschiedenen Produktionsverhältnisse implizieren nun jeweils verschiedene Optimierungsstrategien. Eine Maximierung von Profit in einen landwirtschaftlichen Betrieb etwa fuhrt $\mathrm{zu}$ durchaus anderen Investitionsentscheidungen als eine Maximierung von Einkommen, denn das größtmögliche Einkommen mag unter Umständen eine sehr weitgehende Nutzung der eigenen Arbeitskraft und der vorhandenen Ressourcen implizieren, die weit über die Grenze einer sinnvollen Verzinsung des eingesetzten Kapitals hinausgeht. Eine Maximierung von Einkommen bei einfacher Warenproduktion oder bei Lohnarbeit fallt auch nicht zusammen mit der Optimierung verschiedener Gebrauchswerte, wie sie für die Subsistenzproduktion charakteristisch ist.

Die Spezifität jener Mischung, die die Schicht der Ungesicherten charakterisiert, liegt nun darin, daß die Suche nach Sicherheit eindeutig Priorität vor der Einkommensmaximierung erhält, welche zwar durchaus als Ziel vorhanden, jedoch gegenüber der Stabilisierungsorientierung sekundär ist. Scott sprach bei bäuerlichen Produzenten (1976: 29) von dem safety-first-Prinzip. Unter Drohung der physischen Vernichtung wird auch dort die Produktion aufgenommen, wo Produktionsfaktoren unter ihrem gesellschaftlichen Durchschnitt „entlohnt" werden. Die Untergrenze des Einsatzes von Arbeitskraft und Produktionsmitteln ist theoretisch durch das ökonomische Kriterium des Ersatzes der aufgewendeten Produktionsinputs und des Erhalts der Arbeitskraft gegeben, liegt bisweilen jedoch so weit darunter, daß Hungerkrisen die Folge sind.

\section{Der Stellenwert der Subsistenzproduktion}

Eine besondere Rolle für das Uberleben spielt gerade bei diesem Armutspol die Subsistenzproduktion, wie verschiedene Bielefelder Arbeiten (siehe dazu den Sammelband der Arbeitsgruppe Bielefelder Entwicklungssoziologen 1979) hervorgehoben haben. Angesichts der positiven Resonanz, die diese Studien gefunden haben, müssen wir aber vor einer vorschnellen Generalisierung warnen: Die Subsistenzproduktion ist nicht der
Schlïssel schlechthin, um das Uberleben der Ungesicherten zu klären; sie ist vielmehr der Schlüssel zu wesentlichen Differenzen im politischen Verhalten zwischen verschiedenen Typen von Lebensbedingungen in der Nähe des Armutspols in Ländern der Dritten Welt.

Beispiele für besonders große Bedeutung der Subsistenzproduktion bieten Städte wie Cotonou, bei dem man von einer ,urbanisation sans bidonvilles" sprechen kann (Elwert/Sègbènou 1979), und Groß-Jakarta, wo in weitverzweigten kampungs auch städtische Agrarproduktion für den Eigenbedarf noch ins Gewicht fallt (Evers 1982; Evers/Betke/Sundoyo 1982). Für Jakarta haben wir versucht, den Beitrag von Güterproduktion für den eigenen Bedarf (einschließlich „Dienstleistungsproduktion " wie etwa Hausarbeit usw.) zu quantifizieren. Methodisch ist dies nicht unproblematisch, da wir hierbei Schattenpreise unterstellen mußten. Das Problem dabei ist, daß dann, wenn diese Produkte tatsächlich auf dem Markt auftauchen würden, sich diese Preisrelation insgesamt und damit auch die bisher angenommenen Preise verändern würden. Dieses Problem ist aber in erster Linie ein Problem für die Prognose wirtschaftlicher Entwicklungen. Da der methodische „Fehler" im wesentlichen der gleiche ist, läßt die Berechnung dieser Zahlen durchaus einen Vergleich $z$ wischen verschiedenen Gebieten in der Dritten Welt zu. Wir konnten so zeigen, daß die Subsistenzproduktion in der Großstadt Jakarta bei den ärmeren Schichten einen Anteil von durchschnittlich 18 Prozent zum Haushaltseinkommen leistet. Die Höhe des relativen Anteils der Subsistenzproduktion ist außerordentlich deutlich $(r=-0,8)$ negativ mit der absoluten Höhe des Geldeinkommens korreliert, d.h. um so geringer das Einkommen ist, um so größer ist die Bedeutung der Subsistenzproduktion. Jedoch zeigen schon diese Daten bei der allerärmsten Einkommensschicht (3.000 bis 10.000 Rp. pro Monat) eine Tendenz, auf die wir noch zurückkommen müssen: Bei dieser ärmsten Schicht ist die relative Bedeutung der Subsistenzproduktion etwas geringer als bei der direkt darüberliegenden Schicht, für welche die Subsistenzproduktion die größte Bedeutung hat.

Solchen Ergebnissen lassen sich nun etwa die brasilianischen Untersuchungen gegenüberstellen, die zeigen, daß in bestimmten favelas - 
etwa von Recife - die sogenannten Marginalen gerade durch das Fehlen von Subsistenzproduk. tion, die selbst beim Hausbau nur einen relativ geringen Raum einnimmt, gekennzeichnet sind, während vielmehr die Mittelschicht von Beamten und Angestellten ihren Wohlstand wesentlich auch Gebrauchswerten aus der Subsistenzproduktion unter Einbezug der gebrauchswerterzeugenden Lohnarbeit von Haushaltshilfen/em. pregadas (also eine Gebrauchswertproduktion und keine kapitalistische Lohnarbeit) verdankt (Cavalcanti 1978). Der eigene Garten, die Verarbeitung von Lebensmitteln durch Konservieren, Backen usw., die Reparatur durch Eigenarbeit sind dort wesentlich fur einen bestimmten Lebensstandard.

Ausgehend von der Beschreibung von Verhältnissen der nichtmonetären Subvention der Warenproduktion durch die Subsistenzproduktion (d.h. die Ubernahme eines wesentlichen Teils der Reproduktionskosten durch die Subsistenzproduktion, welche z.B. relativ geringe Löhne ermöglicht; siehe Elwert/Wong 1980), glaubten manche Autoren generalisierend, jede Situation niedriger Löhne auf ein derartiges Verhältnis von Subsistenz- und Warenproduktion zurückführen zu können (u.a. Jacobi und NieB 1980; Fröbel/Heinrichs/Kreye 1981: 13). Diese kritisch gemeinte theoretische Generalisierung verschleiert jedoch nur die Unsicherheit der Lebensbedingungen eines Teils der sogenannten Marginalen. Manche, aber nicht jede Situation niedriger Löhne läßt sich auf die von uns beschriebene Ubernahme der Reproduktionskosten durch die Subsistenzproduktion zurückführen. Ebenso können aber auch Lebensstandards, die extrem von den anderen kulturell in einer Gesellschaft gegebenen Standards abweichen, oder verschleierte Reproduktionskrisen (also keine Fortpflanzung oder kein Uberleben der Arbeiter) hierfür Ursache sein.

Ein Vergleich von favelas verschiedener Bewohnerdichte in Brasilien (Wilkens 1982: 112/113) legt nahe, daß die Bedeutung der Subsistenzproduktion zurückgeht, wenn die Bevölkerungskonzentration zunimmt. Dies ist dadurch zu erklären, daß auch die Subsistenzproduktion Ressourcen voraussetzt. In der Situation knapper Ressourcen wird die Subsistenzproduktion nur einen sehr geringen Beitrag zum Uberleben der Armen leisten, und umgekehrt kann eine reichliche Ressourcenausstattung vor allem mit Land, das etwa für eine intensive Gartenlandwirtschaft genutzt werden kann, den ärmsten Pol der Bevölkerung über jene Grenze der Ungesichertheit hinausheben.

\section{Subsistenzproduktion und Geldeinkommen bei der ärmeren Bevölkerungshälfte}

Anhand von vier Schemata wollen wir verschiedene Typen dieses Verhältnisses von Subsistenzproduktion und Geldeinkommen zeigen. Die folgenden Schemata betreffen jeweils die beiden dem Gesamteinkommen nach ärmeren Quartile der Bevölkerung. Wir stellen hierzu das Geldeinkommen zu einem Subsistenzproduktionsindex (Evers 1981) in Beziehung, der den Prozentanteil von Güterherstellung und Dienstleistungen für den Eigenbedarf am gesamten Haushaltseinkommen mißt.

SCHEMA 1: Agrarischer Bereich mit guter Ressourcenausstattung und relativ egalitärer Verteilung

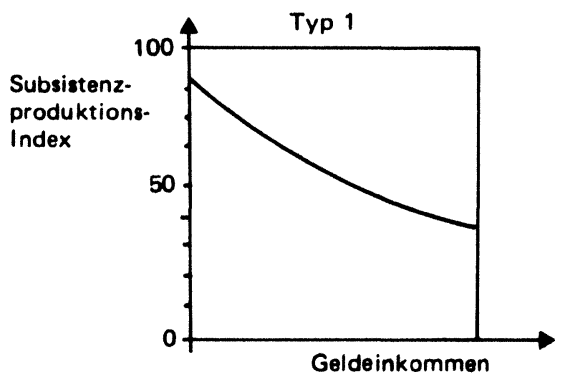

Im Schema 1 sehen wir eine außerordentlich hohe Bedeutung der Subsistenzproduktion. Ganz ohne den Input gekaufter Güter kommt niemand aus (zumindest Salz und Petroleum werden gekauft), doch ist auch noch bei dem bessergestellten Quartil die Bedeutung der Subsistenzproduktion beträchtlich. Insgesamt gilt: Je größer das Geldeinkommen, desto geringer die Bedeutung der Subsistenzproduktion. Ein Beispiel hierfür bietet das von uns untersuchte Dorf Ayou in Bénin (Elwert/Wong 1979) in den siebziger Jahren, wo Bauern mit sehr geringen Geldeinkommen - meist Leute mit vielen noch nicht arbeitsfähigen Kindern - fast die gesamte Ernte zur Ernährung der Familie aufwenden müssen. Wer nach Abzug der Nahrung mehr übrig hat, kann dies verkaufen (z.T. aber auch als Hilfe abgeben) und hat so ein größeres Geldeinkommen. Lohn- 
SCHEMA 2: Ländliches Gebiet mit relativ starker Warenproduktion oder städtisches Gebiet mit relativem Landreichtum

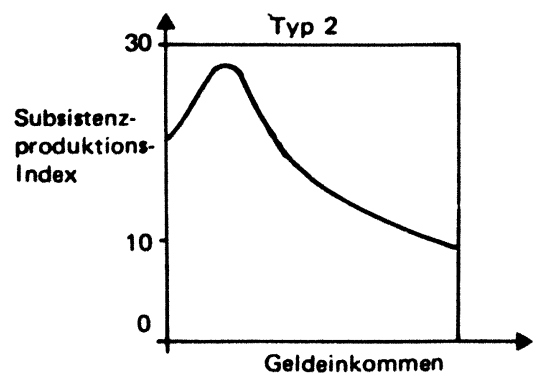

arbeit spielt hier fast keine Rolle. Bei den nach Geldeinkommen ,ärmsten" Haushalten sind auch die Möglichkeiten der Frauen, Handel zu treiben, eingeschränkt.

Die Kurve auf dem zweiten Schaubild entspricht etwa unserem Jakarta-Material (Evers $1981 \mathrm{~b}$ und c). Diese Verteilung ergibt bei der Berechnung eine deutlich negative Korrelation. Bei steigendem Geldeinkommen scheint zunächst generell die Bedeutung der Subsistenzproduktion zu sin$\mathrm{ken}^{8}$. Allerdings weist die in Surveys meist nur mit einer sehr kleinen Zahl vertretene unterste Schicht der Bevölkerung einen geringeren Subsistenzproduktionsindex auf. Dieser Typ 2 kann daher leicht mit dem Typ 1 verwechselt werden, da bei Erhebungen die unterste (wie übrigens auch die oberste) Einkommensschicht oft gar nicht oder zumindest unterrepräsentiert ist: Der aus Kartons gebaute Verschlag, in dem eine alte Witwe ohne Angehörige wohnt, wird leicht übersehen; die infolge der Hungersnot zugewanderten Fulbe-Hirten werden bei der Dorfstatistik nicht mitgezählt - sie tauchen höchstens bei der Aufzählung des nichtmenschlichen Inventars unter den Tieren auf (Busacker/Obbelode 1983: 138); Bettler, die auf Trottoirs übernachten, werden nicht als ansässig betrachtet.

Diesen dritten Typ finden wir vor allem dort, wo erst gekaufte Ressourcen eine sinnvolle Subsistenzproduktion ermöglichen. Diana Wong (1982) beschrieb dies etwa für malaysische Bauern, bei denen erhebliche Subsistenzproduk*

8 Selbst wenn, in absoluten Ziffern berechnet, die Bedeutung dieses Beitrags bei allen Einkommensschichten etwa gleich wäre, würden die hier dargestellten Kurven nach rechts absinken, weil sie den relativen Beitrag der Subsistenzproduktion zum Gesamteinkommen darstellten.
SCHEMA 3: Mittelbäuerliche Subsistenzproduktion bzw. urbane Subsistenzproduktion stabilisierter Proletarier

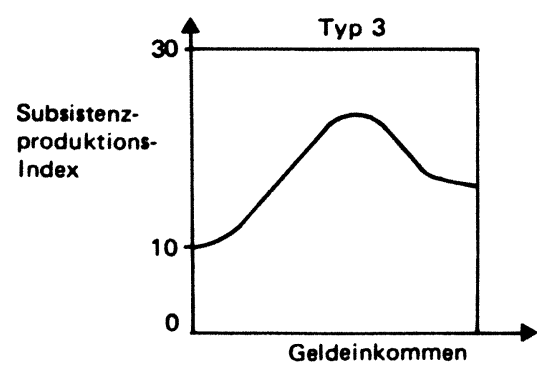

tion erst für die relativ gesicherte Schicht der Mittelbauern (durch double-cropping und Einsatz von Traktoren) möglich wird. Die ärmsten Bauern müssen sofort nach der Ernte alles verkaufen, was sie produzieren und müssen sich später sämtliche Konsumgüter, zum großen Teil auch die Nahrung, kaufen. Im urbanen Bereich, etwa Afrikas, finden wir die Lebenssituation von Arbeitern der Staatsbetriebe oder großer ausländischer Firmen, die mit Hilfe ihres Arbeitgebers oder dank ihres hohen und stabilen Einkommens sich große Parzellen kaufen konnten, auf denen sie fast sämtliches Gemüse, alle Gewürze und den größten Teil des Fleisches, das sie konsumieren, selbst produzieren können. Hierzu gehört eine charakteristische geschlechtliche Arbeitsteilung: Die Frau hat als Hausfrau diesen Bereich zu betreuen, hat also weniger Chancen, entweder selbst Lohnarbeiterin oder einfache Warenproduzentin zu sein.

SCHEMA 4: Ressourcenknappheit, relativ geringe Subsistenzproduktion

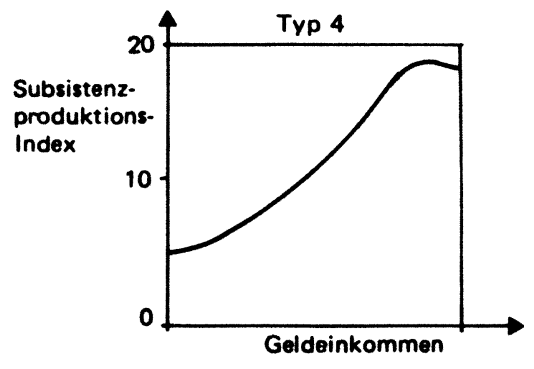

In Situationen der geringen Ausstattung mit Ressourcen, vor allem Land, finden wir den Typ 4, bei dem die Subsistenzproduktion eher ein Charakteristikum der Bessergestellten ist. Erst bei einem relativ hohen Einkommensniveau kann man für den eigenen Bedarf produzieren. Ein Beispiel aus dem ländlichen Bereich sind 
manche Plantagensysteme (s. z.B. Ulshöfer 1981), bei denen der Bodensatz der ärmsten instabilen - Aibeitskräfte die Wohnung mietet, die Nahrung - u.U. sogar schon gekocht - kauft und nicht einmal einen Garten anlegen kann. Die besser situierten Arbeitskräfte können Land zur Nutzung erhalten. Das - männliche Führungspersonal kann die Gattin zur Subsistenzproduktion in Garten, Küche und Nähstube vollständig freistellen.

Die Oasenökonomie des Oman ergibt eine ähnliche Situation: Landlose arbeiten als Lohnarbeiter in der Ólindustrie, während mittlere Bauern auf ihrem bewässerten Land zumindest teilweise für den Eigenbedarf produzieren (Bierschenk 1982).

Die Quantifizierung der „Dienstleistungsproduktion "als Teil des Gesamteinkommens der Haushalte ist besonders schwierig. Die quantifizierte Darstellung der Dienstleistungen innerhalb der Subsistenzproduktion durch Schattenpreise kann vor allem bei niedrigen Werten zu der Mißdeutung Anlaß geben, diese Arbeit könne vernachlässigt werden. Charakteristischerweise hat aber die physische Reproduktion - also Frauenarbeit - nur sehr selten die Warenform und läßt sich deshalb kaum durch Schattenpreise darstellen; wie überhaupt die Frauenarbeit durch die meist zu unterstellenden niedrigen Löhne nur schlecht durch diese Berechnung repräsentiert wird. Die haushaltsinternen Dienstleistungen lassen sich besser anhand der aufgewendeten Arbeitszeit erfassen. Hier werden jedoch oft Merkmale dem sogenannten informellen Sektor als charakteristisch zugesprochen, die tatsächlich für alle Bereiche der Ökonomie gelten.

Minge-Klevana (1980) zeigte, daß mit dem Ubergang von der agrarischen zur industriellen und postindustriellen Gesellschaft die außerhalb des Hauses verbrachte Arbeitszeit sinkt, die Arbeitszeit innerhalb des Hauses aber nicht nur relativ, sondern auch absolut steigt. Dies schlägt sich vor allem als eine besondere Belastung der Frauen nieder (vgl. hierzu Werlhof 1982). Die von MingeKlevana zitierten Studien haben allerdings einen bias für "normale" industriegesellschaftliche $\mathrm{Si}$ tuationen. Dort scheint sich vor allem die Scholarisierung der Kinder als Verlust von kindlicher Arbeitskraft und zusätzliche Belastung für die Hausfrau niederzuschlagen. In der Schicht der
Ungesicherten hat die Schulpflicht nicht diese Bedeutung. Eher finden wir gegenläufig eine zunehmende Bedeutung der Kinderarbeit (vgl. hierzu White 1980).

Die Möglichkeiten zu einer Subsistenzproduktion von Gütern (weniger von Dienstleistungen) sind entscheidend für die Differenzierungen im politischen und ökonomischen Verhalten bei den Ungesicherten. Dort wo praktisch keine Subsistenzproduktion besteht, ist die geographische Mobilität außerordentlich hoch. Es fallt z.B. leichter, den Arbeitsplatz je nach Lohnangebot $\mathrm{zu}$ wechseln, auch wenn man in eine andere Gegend umziehen muß. Zunehmende Urbanisierung, insbesondere die Umsiedlung in Wohnsilos, hat in Singapore die Subsistenzproduktion gesenkt, war aber gleichzeitig mit sehr starkem Arbeitsplatzwechsel (job hopping) verbunden (siehe dazu die Werftarbeiterstudie von Noeleen Heyzer 1973).

Der häufig geäußerten Vermutung, diese Leute „hätten nichts als ihre Ketten zu verlieren" und seien deswegen besonders leicht mobilisierbar für revolutionäre Aktivitäten, stehen wir ähnlich wie Perlman (1976) skeptisch gegenüber. Uns scheint eher, daß eine relativ starke Subsistenzproduktion einen besseren Rückhalt bietet, um Kampfperioden (so auch Einkommenslosigkeit durch Streik) zu überstehen. Die Verteidigung dieser spezifischen Subsistenzbasis ist unseres Erachtens ein Kernpunkt zahlreicher politischer Konflikte (vgl. zu den Kämpfen südostasiatischer Bauern Scott 1976, Hartmann 1981; zu städtischen Kämpfen T. Evers et al. 1979).

Ebenso wichtig sind aber auch die Verteilungsverhältnisse zwischen Männern und Frauen und zwischen Alten und Jungen. Diese Verteilungsverhältnisse können nämlich je nach ihrer Struktur Raum für haushaltsinterne Verteilungskonflikte vor den ,großen" politisch-ökonomischen Konflikten lassen, oder sie können die ökonomische Situation eines Einkommensempfängers innerhalb der Familie als Problem fur alle generalisieren und somit aus der Betroffenheit einer Teilgruppe zu einer generellen Mobilisierung führen.

Während die gesicherte Schicht kooperative oder korporative Sicherungen institutioneller Form hat, ist die Schicht der Ungesicherten nicht etwa 
durch Resignation gekennzeichnet, sondern durch eine aktive Suche nach Sicherheit ${ }^{9}$. Bei dieser Suche nach Sicherheit sticht gerade der Versuch der Schaffung kooperativer Strukturen und der meist vergebliche Appell an den Staat zur Bereitstellung korporativer Strukturen der Sicherung hervor.

Solche Versuche der Sicherung durch kooperative Strukturen werden oft von den Forschern aber nicht als solche erkannt. Sie haben bisweilen die Form von ethnischen Vereinigungen von Personen gleicher Herkunft oder auch die Form ,neuer Kirchen" oder alter Glaubensgemeinschaften mit hoher Kohäsion. Ebenso ist hier auch der Brauch der Gevatterschaft (Patenschaft, compadrio usw.) zu sehen, durch welchen para-verwandtschaftliche Bande geknüpft werden. All diesen Strukturen ist gemeinsam, daß sie wesentlich auch der sozialen Sicherheit ihrer Mitglieder dienen.

Sehr häufig werden solche kooperativen Strukturen der Selbsthilfe als ein Import aus dem ländlichen Bereich betrachtet; ,peasants in the cities“ lautet das Schlagwort. Der Schein trügt jedoch meist. Der Prozeß der Migration vom Land in die Stadt, aber vorher schon die Kommodifikation (warenökonomische Auflösung) der traditionellen bäuerlichen Wirtschaft, bringt Individualisierung und die Auflösung der traditionellen Kooperations- und Solidaritätsformen mit sich. Was wir in den Einwanderungszonen der Städte erleben, ist das heftige Bemühen um eine Neuschöpfung solcher Strukturen im Kontext der Ungesichertheit am Armutspol der Gesellschaft. Das Bewußtsein ethnischer Identität ist so häufig ein Produkt des Wanderungsprozesses in die Stadt (vgl. hierzu Hart 1973, Wallerstein 1960); städtische Klassenkämpfe werden häufig in das Idiom von Rassenkonflikten über-

9 Die ökonomische Situation der Ungesicherten konditioniert dabei das politische Verhalten. Wer die Ressourcen für eine entsprechende Eigenproduktion hat oder wer ein ausreichendes Geldeinkommen hat, kann seinen Bedarf an bestimmten Gütern durch Eigenproduktion oder als Nachfrage auf dem allgemeinen Gütermarkt artikulieren. Wenn diese Möglichkeiten fehlen, weil jemand z.B. kein Land für Eigenproduktion hat, ein zu geringes Geldeinkommen hat oder weil der Gütermarkt durch eine Reservierung verschlossen ist, der kann Nachfrage nur noch als politischen Protest äußern, er muß auf die Straße gehen. setzt (Evers 1975); oft wird eine ethnische Identität erst mühsam konstruiert (vgl. z.B. die North Kavirondo, die sich als ethnische Vereinigung erst in Nairobi konstituierten (Hake 1977: 229) oder die Schaffung konsolidierter Ethnien in Singapore (Benjamin 1977)).

Eine von uns beschriebene Fischergruppe in der afrikanischen Stadt Cotonou (Elwert und Sègbènou 1979) scheint demgegenüber einen Beleg für den Import traditioneller Strukturen zu geben. Diese Fischer zogen in die Stadt als Verwandtschaftsgruppe, fanden dort zum Teil Lohnarbeit, führten aber als Haupterwerbstätigkeit die Fischerei weiter, wobei die Fischerei streng egalitär nach Bootsgemeinschaften und entsprechenden Kollektiven von Aufkäuferinnen organisiert war. Der Name dieser Gemeinschaften ist jedoch schon verräterisch: Sie heißen kompeni vom Englischen ,company". Die Fischer gaben sich, wie sie selbst erzählen, diese Struktur als Reaktion auf die kapitalistische Fischerei, wie sie sich überall an der westafrikanischen Küste entwickelte (vgl. etwa Vercruijsse 1979).

Insbesondere die Händler sind zur Ausübung ihres Berufes auf die Etablierung eines Regelsystems angewiesen. Märkte und Straßenhandel sind planvoll organisiert; Reviere sind abgesteckt und Wettbewerbsregeln festgelegt. Hinzu kommt eine Solidarität der materiellen Unterstützung im Krisenfalle und der politischen Unterstützung gegen die Polizei. Diese Solidarität vermag in der Regel nicht, den Uberlebensbedingungen eine zureichende Sicherung zu geben, wohl aber ist sie wichtig, weil die Versagung der Solidarität ein starkes Sanktionsmittel ist. Das vielbenutzte Bild eines gewaltigen Durcheinanders und bunten Treibens zur Charakterisierung von Märkten und Straßenhandel ist deshalb zu oberflächlich (Wilkens 1982: 92). Diese SelbsthilfeStrukturen sind aber nicht notwendigerweise auch egalitär. Gerade hinter der Erscheinungsform einer Gevatterschaft (compadrio) oder einer religiösen Bruderschaft (cofradia) können sich sowohl bewußt defensiv organisierte egalitäre Strukturen als auch eine kaum verhüllte klientelistische Hierarchie verbergen (vgl. hierzu Celestino und Meyers 1981 und Bennholdt. Thomsen 1976).

Es wäre nun aber eine unzulässige Verallgemeinerung, wollten wir die Suche nach Sicherheit 
als ausschließliches Merkmal der Ungesicherten hervorheben. Auch im zentral-kapitalistischen Bereich finden wir Sicherheitsstrategien, vor allem die Stabilisierung von Märkten durch Bildung von Monopolen und Kartellen, welche im folgenden zur Erklärung des ökonomischen Phänomens der Peripherisierung wichtig werden wird.

\section{Peripherisierung auf der ökonomischen Ebene}

Es ist mittlerweile selbstverständlich geworden, auf der Weltmarktebene von Zentrum und Peripherie zu sprechen ${ }^{10}$. Auf nationaler Ebene läßt sich empirisch analog zur Weltsituation ein ähnlicher Prozeß der Differenzierung feststellen. Wir sprechen hier bewußt von einem Prozeß, denn es scheint uns nicht mehr haltbar zu sein, „duale" Strukturen als etwas Konstituiertes aufzufassen, was schon als Handikap dem Entwicklungs-/UnterentwicklungsprozeB von Anfang an mitgegeben war. Vielmehr konstituieren sich der Zentrums- und der Peripheriepol als Spannungsverhältnis im Rahmen spezifischer Marktdynamiken im Zuge der Kommodifikation und der Durchsetzung staatlicher Wirtschaftslenkung.

Genau genommen haben wir es in dem Wirtschaftsbereich, der am ehesten dem sozialen Bereich der Ungesicherten entspricht, mit einem Ressourcenmangel zu tun, wie er eigentlich nach den Gesetzmäßigkeiten einer Marktwirtschaft nicht auftreten dürfte.

Myint formulierte dies schon 1971 als Quintessenz seiner ökonomischen Untersuchungen (315-316):

". . the most significant aspect of economic dualism as it exists in the present-day underdeveloped countries is that scarce inputs such as capital funds, foreign exchange and public economic facilities, notably transport and communications and power, are being made available on excessively favourable terms to the larger units in the modern sector including the public sector, and on excessively unfavourable terms to the small economic units in the traditional sector."

10 Wir halten es allerdings nicht für sinnvoll - wozu Wallerstein zu neigen scheint - Zentrum und Peripherie (und eventuell noch Semi-Peripherie) als Räume abzugrenzen. Weitaus sinnvoller scheint uns methodologisch eine andere Art von Typologie, bei der zwei Extreme sich gegenüberstehen.
Ressourcenmangel wird hier in einem spezifischen Sinn verstanden, also nicht nur der Mangel an Betriebsvermögen (Unterkapitalisierung), sondern auch der Mangel an Infrastruktur-Investitionen, staatlichen Dienstleistungen und an Kreditmöglichkeiten ist gemeint. Bei Infrastruktur-Investitionen ist insbesondere an Straßen, Schienenwege, Strom- und Wasserzufuhr zu denken. Die staatlichen Dienstleistungen werden in ihrer Bedeutung besonders unterschätzt (vgl. Augel 1979). Wir haben hier unter anderem die Gewährung gewaltfreier Räume und weitere Bereiche des Justizwesens im Auge (vgl. auch Waldmann 1980). Wenn eine Firma keine Chancen hat, die Schulden von einem Kunden einzuklagen, ist ihre Lebensfähigkeit sehr behindert; wenn Räuber den Gütertransport bedrohen, erstirbt der Handel.

Dieser Mangel betrifft nun nicht schlechthin alle Arbeit der Ungesicherten. Er betrifft im wesentlichen die einfache Warenproduktion und die Subsistenzproduktion von Ungesicherten. Habitat-Produktion besteht ja nicht schlicht darin, ein Haus hinzustellen, auch ein System der Wasserzufuhr und der Entwässerung ist notwendig. Die Lohnarbeit von Ungesicherten ist von der Reproduktionsseite immer von dieser Unterkapitalisierung im weiteren Sinne betroffen; von der Produktionsseite her gilt dies nicht immer. Es gibt allerdings auch Extremfalle, wo sogar der kleinkapitalistische Unternehmer der Schicht der Ungesicherten zuzurechnen ist. Hier erfaßt Unterkapitalisierung auch einen Teil der kapitalistischen Betriebe (dies gilt sogar für Hong Kong).

Inwiefern ist diese Unterversorgung nun ein Zuwenig und nicht nur ein ,Wenig" an Kapital, woran wird der relative Mangel gemessen? Die knappe Ausstattung mit Infrastrukturkapital, Investivkapital und Krediten widerspricht dem zum Teil meßbaren wirtschaftlichen Po-. tential der Peripherie der jeweiligen Okonomien. Diese Benachteiligung bei der Ressourcenverteilung entspricht nicht der "Logik" einer Marktwirtschaft.

Am deutlichsten zeigt Myint diese Unterversorgung am Kapitalmarkt, wo hohe Zinssätze einerseits einen eklatanten Uberschuß der Nachfrage nach Kapital zeigen und die Tatsache, dafs die Zinsen auch tatsächlich regelhaft von der Wirtschaft dieses Bereichs aufgebracht werden kön- 
nen, die Ertragskraft unter Beweis stellt. Die vorgeblichen intrinsischen Merkmale der informellen Ökonomie, wie hohe Arbeitsintensität und verwandte technologische Merkmale, können als rationales ökonomisches Verhalten aus den Faktoren der hohen Zinssätze und niedrigen Löhne abgeleitet werden (Myint 1971: 324-331).

Durch die nicht marktadäquate Allokation des Kapitals fließen stattdessen die Gelder in den staatlichen Bereich sowie in den Bereich der Groß- und Monopolunternehmen und der Kartelle. Kapitalismus darf nicht automatisch mit Marktwirtschaft gleichgesetzt werden. Die optimale Allokation von Ressourcen durch Marktmechanismen ist eine der Möglichkeiten zur Kapitalakkumulation. Mit dem Einsatz von Macht zur Veränderung des Marktgeschehens (Absprachen, Einsatz staatlicher Macht) ist eine weitere Möglichkeit zur Akkumulation gegeben. Bereits Max Weber beschrieb ,voluntaristische Marktregulierungen“" (1956: 43). Wir bezeichnen hier diese Transformation der Marktstruktur als eine Ver-macht-ung des Marktes.

Ein Teil der OKonomie wird so „peripherisiert“ (vgl. hierzu das Beispiel der modernen türkischen Wirtschaftsgeschichte bei Keyder 1970). Dieser Prozeß der Peripherisierung - also nicht eine vorgegebenen Struktur - ist das eigentliche Phänomen. Nicht Traditionalismus, mangelndes Erwerbsstreben oder ähnliche Merkmale der Ungesicherten, sondern Monopolisierungs- und Kartellisierungsprozesse sind die Ursachen der Unterkapitalisierung und damit der Peripherisierung ganzer Wirtschaftsbereiche.

Dieser Prozeß der Peripherisierung hat zwar zum einen unbestreitbar eine marktmäßige Verursachung. Es ist dies die Tendenz der Eliminierung der nichtkonkurrenzfähigen Kapitale. Das bedeutet aber nicht, daß ein Betrieb, der als kapitalistischer Betrieb ausgeschieden ist, gänzlich aus der Sphäre der Produktion verschwinden muß. Es existieren gerade in der Dritten Welt eine hohe Anzahl von Betrieben, die ihren Mitarbeitern lediglich die Reproduktion sichern sollen, die oft nur zu diesem Zweck gegründet sind. Zum anderen aber erfolgt die Unterdrükkung dieser Produktionsformen durch feststellbare Vermachtungsstrukturen der bereits etablierten kapitalistischen OKonomie und des inter- venierenden Staatsapparates. Der erschwerte Zugang zu Krediten und Subventionen, das unzureichende System der Rechtssicherheit, die mangelnde infrastrukturelle Versorgung und repressive behördliche Auflagen stehen als Beispiele für staatlich und parastaatlich verursachte defizitäre Beeinflussungen. In weiten Bereichen kommt es darüber hinaus zu voluntaristischen Marktregulierungen. Monopolisierung und Kartellisierung bieten neben der ökonomischen Macht (Dumpingpreise, höhere Produktivität) zusätzliche Mittel, Märkte und den Zugang zu ihnen zu beeinflussen (Bestechung, Werbung).

Die strukturellen Defizite und die Deformierung des Marktes durch Macht, die als Widerstände gegen den Aufbau einer marktfähigen Produktion wirken, bleiben natülich nicht ohne Antwort. Aus sich heraus ist die informelle Produktion gezwungen, ihr gangbare Alternativen aufzunehmen, um die Anbindung an den Markt vollziehen zu können. Folgende Mittel zeichnen sich dabei als Strategie ab:

- Senkung der Produktionspreise durch Verbilligung des konstanten Kapitals, z.B. durch Aufnahme der Produktion in Wohnräumen, auf der Straße etc.; Verzicht auf teure Maschinen, Energieeinsatz (dafür einfache Geräte, tierische oder menschliche Antriebskraft).

- Das variable Kapital wird verbilligt, indem nicht auf Lohnarbeitskräfte zurückgegriffen wird, sondern oft Familienmitglieder oder Verwandte produzieren. Auch die partnerschaftliche Produktion von Kompagnons ist ein solcher Weg.

- Wenn freie Ressourcen als Produktionsgrundlage erschöpft sind, findet man oft billige Substitute als Basis. Auch hier liegt eine erzwungene Beschränkung der Kapitalauslagen vor. Besonders im Kunsthandwerk zeigt sich die Präferenz für Materialien, die geeignet sind, verhältnismäßig große Mengen an Arbeitskraft aufzusaugen.

- Die Kreditaufnahme, wo immer sie nötig wird, muß den Weg der persönlichen Kreditvermittlung gehen, oft begleitet mit hohen Zinsraten, die sich ökonomisch aus hohen Risiken (keine materielle Sicherheit) herleiten, zusätzlich aber eine Verzerrung durch asymmetrische Marktmacht zugunsten des Kreditgebers enthalten. 
Die Marktanbindung der informellen Produzenten hat sozusagen ,um jeden Preis" stattzufinden, denn anders als beim kapitalistischen Un. ternehmen ist bei einem nicht realisierten Produkt sofort die Reproduktionsbasis geschmälert.

Dieses Phänomen der nicht-marktmäßigen Allokation von Ressourcen ist nun auch für die zentral-kapitalistischen Länder nichts Ungewöhnliches. Nur finden sich hier die Entsprechungen nicht unbedingt in der Situation jenes Bereichs, der in vorschneller Analogiebildung mit dem sogenannten informellen Sektor der Dritten Welt gleichgestellt wird, also jener Mischung aus doit-yourself, Schwarzarbeit und Hausarbeit.

Beispiele sind vielmehr das Verhältnis von Konferenzlinien der Schiffahrtskonfer enzen gegenüber der Tramp-Schiffahrt oder das Verhältnis von Markenbenzin-Vertriebsnetzen einerseits und dem Spot-Markt und den freien Tankstellen andererseits.

Das Problem, das sich hier den Kapitaleignem stellt, ist das von fluktuierenden Märkten. Um sinnvoll investieren zu können, streben sie eine Beruhigung des Marktgeschehens an. Dies erreichen sie durch derartige Kartellisierungen. Der Preis - den sie allerdings nicht selbst zahlen müssen - ist eine desto größere Fluktuation im verbleibenden Restmarkt. Der freie Markt, der sich um den kartellisierten Zentralbereich herumlagert, trägt das Expansions- und Kontraktionsrisiko. Die Investitionen konzentrieren sich auf das „Rückgrat" dieser Branche in der kartellisierten Mitte. „Der harte Kern“ muß die Integration der Randbereiche verhindern, damit er nicht selbst derart expandiert, daß Schwankungen des Marktgeschehens auf seine Investitionsmöglichkeiten durchschlagen, d.h. er selbst unsicher wird.

Dabei darf nicht übersehen werden, daß bei Krisen in der Industriegesellschaft, wenn der peripherisierte Bereich zunimmt, bzw. in Entwicklungsgesellschaften durchweg dieser Bereich die Produktionsstruktur des harten Kerns beeinflussen kann. So wurden in Recife/Brasilien zwar nur $2 \%$ des Haushaltseinkommens für Hausgehilfinnen und Dienstleistungen im Haushalt ausgegeben, aufgrund der geringen Lohnsumme damit aber der Absatz von Waschmaschinen, Küchengeräten und dergl. praktisch verhindert (Cavalcanti 1978: 58).
Wir haben zur Analyse der Schicht der Ungesicherten, also einer auf der Handlungsebene abgrenzbaren Sozialkategorie, die Darstellung des mit ihr zusammenhängenden ökonomischen Phänomens der Peripherisierung eines Teils der Wirtschaftssubjekte gestellt, nicht um das Bild abzurunden - dazu fehlt noch viel - sondern um auf einen notwendigen analytischen Schritt hinzuweisen: Die Analyse des peripherisierten Teils der Okonomie ist ohne die Analyse der Vermachtung der Gesamtökonomie nicht zu leisten. Damit sprechen wir keineswegs nur die Analyse von Peripherisierungsprozessen in der Dritten Welt an, sondern möchten eine allgemeine analytische Maxime formulieren, die auch für die entwickelten Industrienationen gilt.

\section{Mangelnde Kohäsion}

Für die Situation der Ungesicherten ist die Schwäche - oft sogar das Fehlen - einer kooperativen Organisation ausschlaggebend. Diese Schwäche ist wesentlich auch durch eine mangelnde Kohäsion bedingt. Kohäsion ist jede Wir-Gruppen konstituierende Interaktion (also auch in nachbarschaftlicher Kooperation, kirchlichen Gruppen, politischen Geheimbünden usw.).

Andererseits ist die Peripherisierung eines Teils der Ökonomie ein Hindernis, um überhaupt korporative Strukturen zu schaffen; zur Peripherisierung gehört eben, daB nicht in die Schaffung korporativer Institutionen investiert wird. Das Fehlen von korporativen Strukturen ergibt sich schon logisch als Implikat der Nicht-Formalisierung dieses Bereichs durch den Staat.

Den Versuchen, kooperative Strukturen wie Gewerkschaften zu schaffen, steht entgegen, $\mathrm{da} B$ es an den Rücklagen mangelt, die eine langgezogene politische Konfrontation ermöglichen würden. Die mangelnde Stärke der vorhandenen kooperativen Strukturen impliziert, $\mathrm{da} B$ keine pressure groups vorhanden sind, die eine Formalisierung des ökonomischen Lebensraums der Ungesicherten erfordern oder erzwingen könnten. Die entscheidende Machtquelle, die die Ungesicherten potentiell zur Hand haben, nämlich soziale Kohäsion (Elias/ Scotson 1965), ist das Ziel der zahlreichen Organisationsversuche, die auf der Handlungsebene der deutlichste Ausdruck der von uns 
postulierten Suche nach Sicherheit ist. Diese Versuche, Macht zu erlangen, sind in der Regel erfolglos. Eine Emanzipation der ungesicherten Proletarier, einfachen Warenproduzenten und Bauern zum Status des Gesicherten (nur Emanzipation, nicht Revolution genannt, weil die Produktionsverhältnisse selbst nicht geändert werden) findet nur in seltenen Fällen statt. Dies widerspricht eklatant den Omnipotenz-Phantasien einiger kritischer Entwicklungswissenschaftler, die in den Slumbewohnern ein revolutionäres Potential sehen wollen. Dann, wenn Selbsthilfe-Initiativen die notwendige Kohäsion schaffen, findet eine ,Formalisierung“ durch den Staat statt ${ }^{11}$.

Daß, wie oben gesagt, die Peripherisierung eines Teils der Okonomie auch ein Hindernis ist, um uberhaupt kooperative Strukturen zu schaffen, gilt besonders dann, wenn die Subsistenzproduktion keine oder nur eine schwache Rolle zur Stabilisierung der Bedingungen des Uberlebens spielt. Wer von der Hand in den Mund lebt, kann keine Gewerkschaftsbeiträge zahlen.

Es zeigt sich also, daß erstens die mangelnde soziale Kohäsion und zweitens die Peripherisierung sich in ihrer Wirkung gegenseitig verstärken und als Endprodukt einen selbst nur unscharf abgrenzbaren, aber doch deutlich erkennbaren Bereich von relativer Armut konstituieren ${ }^{12}$. Die (erfolglose) Suche nach Sicherheit und das Streben nach Macht durch Kohäsion auf der Ebene der sozialen Prozesse einerseits und die Vermachtung und dadurch Peripherisierung auf der Ebene der ökonomischen

11 Vgl. zu Nairobi die Darstellung der ,,self-help city“ von Hake (1977), die sich auf den Zeitraum bis 1972 bezieht und die neuere Darstellung von Chege (1981), die implizit das Produkt eines Prozesses der politischen Anerkennung darstellt.

12 Diese Mechanismen können 2.T. auch dazu dienen, Prognosen zu bilden. Während die Kohäsion langfristig auch den Dynamiken von sozialen Bewegungen unterliegt und durch die damit implizierte Bindung an transzendentale Hoffnungsorientierung sich jeder auf ökonomistisch aufzählbaren Daten beruhenden Prognose entziehen muß, können wir bezüglich der Peripherisierung prognostizieren, daß mit zunehmender Fluktuation der Märkte auch in anderen Bereichen - nicht nur der Dritten Welt - die Stabilisierung eines Kernbereichs durch Kartellisierung zu einem derart dominanten Phänomen wird, daß korrelativ hierzu ein Teil der Okonomie zum peripherisierten Pol absinken muß.
Prozesse andererseits konstituieren im Zusammenwirken zwei teilweise kongruente Bereiche: die Schicht der Ungesicherten bezogen auf die Lebenssituation der Menschen und die Peripherisierung bezogen auf die ökonomische Struktur.

\section{Literatur:}

Arbeitsgruppe Bielefelder Entwicklungssoziologen (Hrsg.), 1981: Subsistenzproduktion und Akkumulation. Bielefelder Studien zur Entwicklungssoziologie. Bd. 5. 2. Aufl. Saarbrücken: Verlag Breitenbach.

Armstrong, W.R./McGee, T.G., 1980: A theory of urban involution. S. 228-234 in: H.-D. Evers (Hrsg.), Sociology of Southeast Asia. Kuala Lumpur: Oxford University Press.

Augel, Johannes, 1982: Contribution of Public Goods to Household Production - Case Study from Brazil. Arbeitspapier. Universität Bielefeld, Fakultät für Soziologie.

Bennholdt-Thomsen, Veronika, 1976: Zur Bestimmung des Indio. Indiana, Beiheft 6. Berlin.

Benjamin, Geoffrey, 1976: The cultural logic of Singapore's ,multiracialism'. S. 115-133 in: R. Hassan (Hrsg.), Singapore: Society in Transition. Kuala Lumpur.

Berger, Johannes, 1982: Zur Zukunft der Dualwirtschaft. S. 97-117 in: Benseler, F./R.G. Heinze/ A. Klönne (Hrsg.): Zukunft der Arbeit. Hamburg: VFA.

Bierschenk, Thomas, 1983: Weltmarkt, Stammesgesellschaft und Staatsformation in Südostarabien (Sultanat Oman). Diss. Fakultät für Soziologie, Bielefeld.

Boeke, Jan H., 1980: Dualism in colonial sectors. S. 26-37 in: H.-D. Evers (Hrsg.), Sociology of Southeast Asia. Kuala Lumpur: Oxford University Press.

Busacker, Dietrich/Obbelode, Marita, 1983: Imaginäre Befreiung - Untersuchungen zu Selbsthilfe-Gruppen in Obervolta. Diplomarbeit, Fakultät für Soziologie, Bielefeld.

Cavalcanti, Clóvis, 1978: Viabilidade do Setor Informal. Recife: Ministério da Eduçāo e Cultura.

Celestino, Olinda/Meyers, Albert, 1981 : Las cofradias en el Péru: región central. Frankfurt: Vervoert.

Chege, Michael, 1981: Electoral politics in Mathare and Dagoretti. Review of African Political Economy 20: 47-88.

Chesneaux, Jean, 1971: Secret Societies in China. Hong Kong.

Choe, Jae-Hyeon, 1982: Die Reziprozität zwischen dem formellen und informellen Sektor: Eine Interpretation anhand von biographischem Material. Internationales Asienforum 13: 269-286.

Connolly, Priscilla, 1982: Critica del „Sector Informal“" como Concepto Aplicado - con Referencia a la Estructura de la Ciudad de México. Paper, Xth World Congress of Sociology, Mexico City.

Elias, Norbert/Scotson, J., 1965: The Established and the Outsiders. London: Cass. 
Elwert, Georg, 1981: Die Dreierschichtung von Proletariat und kleinen Warenproduzenten in der Dritten Welt. S. 599-604 in: W. Schulte (Hrsg.), Soziologie in der Gesellschaft. Universität Bremen.

Elwert, G., 1982: Probleme der Ausländerintegration. Gesellschaftliche Integration durch Binnenintegration? Arbeitsberichte der Fakultät für Soziologie Nr. 30. Universität Bielefeld.

Elwert, G./Sègbènou, R., 1980: Le village dans la ville - les pêcheurs de Cotonou et l'urbanisation. Vierteljahresberichte - Probleme der Entwicklungsländer Nr. 79, 1980: 41-45.

Elwert, G./Wong, D., 1980: Subsistence production and commodity production in the Third World. Review III, 3: $501-522$.

Evers, Hans-Dieter, 1975: Urbanization and urban conflict in Southeast Asia. Asian Survey 15,9: 775785.

Evers, H.-D., 1980: Subsistence production and the Jakarta .Floating Mass'. Prisma 17: 27-35.

Evers, H.-D., 1981 a: Zur Theorie der urbanen Unterentwicklung. Die Dritte Welt 9: 61-68.

Evers, H.-D., 1981b: The contribution of urban subsistence production to incomes in Jakarta. Bulletin of Indonesian Economic Studies (Australian $\mathrm{Na}$ tional University, Canberra) XVII, No. 2: 89-96.

Evers, H.-D., 1981 c: Subsistence Production and Wage Labour in Jakarta. Arbeitspapier No. 8. Forschungsschwerpunkt Entwicklungssoziologie, Universität Bielefeld.

Evers, H.-D./Betke, F./Clauss, W./Piomo, S./Wong, D., 1982: Die soziale Organisation des Subsistenzbereichs: Untersuchungen über Urbanisierung und soziale Entwicklung in Südostasien (Abschlußbericht an die Stiftung Volkswagenwerk). Bielefeld: FSP Entwicklungssoziologie, Universität Bielefeld.

Evers, Hans-Dieter/Betke, F./Pitomo, S., 1982: Die Komplexität der Grundbedürfnisse. Ms. Bielefeld.

Evers, Tilman/Muller-Plantenberg, C./Spessart, S., 1979: Stadtteilbewegung und Staat. Kämpfe im Reproduktionsbereich in Lateinamerika. S. 118-170 in: Lateinamerika: Analysen und Berichte 3: 118-170.

Fanon, Frantz, 1961: Les damnés de la terre. Paris: Maspero.

Fröbel, F./Heinrichs, J./Kreye, O., 1977: Die neue internationale Arbeitsteilung. Reinbek.

Geertz, Clifford, 1963: Peddler and Princes. Chicago/ London.

Guisinger, Stephan/Irfan, M., 1979: Pakistan's informal sector. The Journal of Development Studies 16: 412-426.

Hake, Andrew, 1977: African Metropolis - Nairobi's Self-Help City. London.

Hart, Keith, 1973: Informal income opportunities and urban employment in Ghana. The Journal of Modern African Studies 11 (1): 61-89.

Hartmann, Jörg, 1981: Subsistenzkrise und Rebellion: Eine Kritik der ,moral economy' bäuerlicher Gesellschaften. Arbeitspapier No. 4. FSP Entwicklungssoziologie, Universität Bielefeld.

Heyzer, Noeleen, 1973: Shipyard Workers in Singapore. M.A. Thesis, University of Singapore.

Jacobi, Carol/NieB, Th., 1980: Hausfrauen, Bauern, Marginalisierte: Uberlebensproduktion in ,Dritter" und ,Erster" Welt. Bielefelder Studien zur Ent- wicklungssoziologie. Bd. 10. Saarbrücken: Verlag Breitenbach.

Keyder, Caglar, 1978: Crodit in ther Process of Peripheral Structuration. Working Paper. Ankara: METU.

King, Ambrose/Man, P., 1974: The Role of Small Factories in Economic Development: The Case of HongKong. Working Paper. Chinese University of Hong Kong.

Kowarick, Lucio, 1982: Marginalidade, Algumas Questóes Teóricas e Empíricas. Paper: Xth World Congress of Sociology, México City.

Maas, Utz, 1982: Versuch einer kulturanalytischen Bestimmung ausländerpädagogischer Aufgaben. In: Müller-Kohlenberg, H. (Hrsg.): Ausländerpädagogik. Osnabrïck: Universität, Arbeitsstelle fưr Ausländerpädagogik.

Mao Tse-tung, 1968 (1926): Analyse der Klassen in dor chinesischen Gesellschaft. In: Mao Tse-tung, Ausgewählte Werke. Bd. 1. Peking.

Minge-Klevana, Wanda, 1980: Does labor time decrease with industrialization? A survey of time-allocation studies. Current Anthropology 21 (3): 279-298.

Moir, Hazel, 1978: Jakarta Informal Sector. Jakarta: Leknas.

Mühlmann, W.E., 1964: Chiliasmus und Nativismus. Berlin.

Myint, Hla, 1971: Economic Theory and Underdeveloped Countries. New York.

Nelson, Joan, 1969: The urban poor - disruption or political integration in Third World Cities. World Politics 22 (1): 393-414.

Olivier, Jean Pierre, 1982: Despotische Ausbeutung und Klassenkämpfe im Songhay/Djerma-Land (Niger). In: Elwert, G./R. Fett (Hrsg.): Afrika zwischen Subsistenzökonomie und Imperialismus. Frankfurt.

Peattic, Lisa/Aldrete-Haas, J., 1981 : „Marginal“ Settlements in Developing Countries: Research, Advocacy of Policy, and Evolution of Programs.

Perlman, Janice, 1976: The Myth of Marginality. Berkeley.

Santos, Milton, 1978: The Shared Space. London/New York.

Scott, James, 1976: The Moral Economy of the Pcasant. New Haven.

Sethuraman, S.V., 1976: The urban informal sector: Concept, measurement and policy. International Labour Review 114 (1): 69-81.

Stauth, Georg, 1982: "Milieus of Reproduction" and „Production Systems". Arbeitspapier zum Seminar "Households and the World-Economy" Maj 1982. FSP Entwicklungssoziologie, Universität Bielefeld.

SUDENE/UFPE, 1976: Abastecimento Alimentar no Nordeste Urbano. Grande Recife: Recife Ministério da Eduçäo e Cultura.

Thompson, Edward P., 1971: The moral economy of the English crowd in the eighteenth century. Past and Present 50: 76-136.

Ulshöfer, Petra, 1922: The Function of Paid and Unpaid Labour in a Plantation Economy: The Case of Guatemala. Paper presented at the XI European Congress for Rural Sociology. Helsinki.

Vercruijsse, Emile, 1979: Class formation in the peasant economy of Southern Ghana. Review of African Political Economy 15/16: 93-104.

Waldmann, Peter, 1980: Unsicherheit als Alltagserfahrung in Lateinamerika. Aus Politik und Zeitgeschichte No. 3. 
Wallerstein, 1., 1960: Ethnicity and National Integration in West Africa. Cahiers d'études africaines No. 3.

Wallerstein, J., 1974: The Modern World System. New York.

Weber, Max, 1953: Wirtschaft und Gesellschaft. Tübingen.

Weeks, John, 1975: Policies for expanding employment in the informal urban sector of developing economies. International Labour Review 113,3: 1-13.

Werlhof, Claudia, v., 1981: Der Proletarier ist tot. Es lebe die Hausfrau? Sterz, Zeitschrift für Literatur (Graz) 18,3: 6-7; 19,4: 24-26.

White, Benjamin, 1980: Child Labour and Population Growth: Some Studies from Rural Asia. Vortrag. FSP Entwicklungssoziologie, Universität Bielefeld.
Wilkens, Werner, 1982: Lohnarbeit, informelle Beschäftigung und Subsistenzproduktion in Nordostbrasilien. Diplomarbeit. Fakultät für Soziologie, Universität Bielefeld.

Wong, Diana, 1982: Case studies of village households. In: Evers, H.D., et al.: Die soziale Organisation des Subsistenzbereichs: Untersuchungen über Urbanisierung und soziale Entwicklung in Südostasien (Abschlußbericht an die Stiftung Volkswagenwerk). Bielefeld: FSP Entwicklungssoziologie, Universität Bielefeld. 